\title{
Positron emission tomography for the evaluation of soft-tissue sarcomas and bone sarcomas
}

\author{
Cristina Nanni • Maria Cristina Marzola • \\ Domenico Rubello • Stefano Fanti
}

Published online: 25 July 2009

(C) Springer-Verlag 2009

\section{Introduction}

In the paper by Charest et al. [1] published in this issue of the European Journal of Nuclear Medicine and Molecular Imaging, the sensitivity of FDG PET/CT in detecting sarcoma lesions turned out to be very high (80 to $100 \%$ according to the histological type of disease). This result, obtained retrospectively on a large series of 212 patients, is concordant to what is reported in the recent literature and suggests one should consider this imaging method in the routine diagnostic work up of patients affected by sarcomas for staging and restaging the disease. Interestingly, FDG PET/CT had a high sensitivity both for bone and soft-tissue sarcoma and only ten false-negative cases were recorded. Some of these false-negatives were caused by the small diameter of the lesions and the remaining ones were related to the $\mathrm{SUV}_{\max }$ cut-off value chosen to discriminate between

This Editorial Commentary refers to the article doi:10.1007/s00259009-1203-0

\section{Nanni $\cdot$ S. Fanti}

Department of Nuclear Medicine,

Azienda Ospedaliero-Universitaria di Bologna,

Policlinico Sant'Orsola-Malpighi,

Bologna, Italy

M. C. Marzola • D. Rubello

Department of Nuclear Medicine,

Santa Maria Della Misericordia Hospital,

Rovigo, Italy

D. Rubello $(\bowtie)$

Department of Radiology, Medical Physics, Nuclear Medicine, Chief of Service Nuclear Medicine, PET/CT Centre,

Santa Maria della Misericordia Hospital,

Viale Tre Martiri 140,

45100 Rovigo, Italy

e-mail: domenico.rubello@libero.it malignant and benign lesions (2.5). Probably the latter ones could have been avoided in the clinical practice taking into consideration also visual criteria and not only SUV parameters. According to the literature, besides staging and restaging, FDG PET/CT could also play an important role for assessing the patient prognosis and the response to chemotherapy.

\section{General aspects of sarcomas}

Soft-tissue sarcomas are a group of neoplasms of mesodermal origin that constitutes approximately $1 \%$ of adult malignancies and $7 \%$ of paediatric malignancies. Bone sarcomas are very rare as well, representing only $0.2 \%$ of all new cancer diagnoses, with a biphasic pattern including a peak in adolescence (primary sarcomas) and a peak in the elderly (secondary sarcomas associated with Paget's disease and irradiated bones).

Most soft-tissue sarcomas are sporadic with no specific etiologic agent. In some cases, however, a predisposing factor can be recognized, such as exposure to alkylating chemotherapeutic agents, Paget's disease, areas of bone infarction, irradiated bones, neurofibromatosis, tuberous sclerosis, Gradner's syndrome and Li Fraumeni syndrome.

While soft-tissue sarcomas arise predominantly in the abdomen and in the extremities, bone sarcomas may arise in any bone and within any region of a given bone. However, osteosarcoma arises most frequently in the long bones of the lower extremities while Ewing's sarcoma has a predilection for the long tubular bones, the flat bones of the pelvis and the ribs.

Usually the clinical presentation of patients with softtissue sarcomas depends on the primary tumour site while most patients with bone sarcoma present with localized pain. The diagnosis of sarcoma arises after a CT scan for 
soft-tissue sarcomas, a MR scan for bone sarcomas and a targeted biopsy, while the disease staging is performed with satisfactory accuracy mainly with CT, especially for the evaluation of lung metastasis.

The prognosis of these neoplasms is strongly related to several factors among which it is important to remember the extent of the disease at diagnosis, the grade of the tumour, the age of the patient, the presence of microscopically positive margins after resection, the presence of metastasis at disease diagnosis and a long duration of symptoms before the diagnosis.

The treatment of sarcomas relies basically on surgery, which is aimed at complete eradication of the disease. However, it is possible to increase patient survival by combining surgery with chemotherapy and radiation therapy (adjuvant or neo-adjuvant), in order to facilitate surgical excision of large tumours or to consolidate local treatment after surgical resection.

Follow-up for patients successfully treated for soft-tissue or bone sarcoma requires an understanding of the relationship between the risk of recurrence and the amount of time elapsed after treatment. Long-term follow-up studies demonstrate that approximately $80 \%$ of patients who develop recurrent disease will do so within the first 3 years after therapy, and that patients who are alive without recurrence at 5 and 10 years after therapy apparently still carry a risk for a subsequent late recurrence [2]. All surveillance strategies include physical examination, appropriate imaging of the primary site of the tumour and chest imaging for the risk of lung metastasis.

\section{Sarcomas and PET}

Since the great part of sarcomas are intermediate or highgrade tumours, PET can be used as an additional and valuable imaging method to integrate the standard diagnostic techniques (mainly CT and MR).

The indications to perform a PET scan in patients affected by sarcomas are multiple. Several papers were published in the last years and, according to the number of articles, it turns out that PET can be successfully used for predicting the prognosis, for staging the disease and for assessing the response to therapy [3]. FDG seems to be the most accurate radiopharmaceutical. However, many other tracers have been tested with positive results, among which the most important are 11C-Choline, 18F-FLT and 11C-Methionine.

\section{PET and the prediction of prognosis}

Because of the generally poor prognosis, it is important to find out as many prognostic factors as possible when evaluating a patient affected by sarcoma, in order to choose the best therapeutic approach and to correctly schedule the follow-up exams.

Many authors analysed the possible role of FDG-PET in predicting the prognosis both when performed at diagnosis and after the pre-surgical chemotherapy.

Eary et al. [4], for example, evaluated a series of 238 patients affected by different kinds of sarcomas who underwent FDG-PET before therapy (chemotherapy or surgical resection) and compared the PET result with the overall survival and the disease-free survival. PET images were analysed in terms of $\mathrm{SUV}_{\max }$ and spatial heterogeneity of FDG distribution within the primary mass, since it was noticed that biologic heterogeneity (including proliferation, necrosis, non-cellular accumulations such as matrix material and fibrous tissue, differences in blood flow, cellular metabolism, oxygenation and receptorial expression) is a very important feature of malignant tumours. The authors found that both $\mathrm{SUV}_{\max }$ (which is an index of malignancy) and FDG heterogeneous distribution can distinguish between higher risk patients and lower risk patients, and that it is therefore possible to recognize two groups of patients with different prognosis on the basis of FDG-PET results.

Similar results were obtained by Lisle et al. [5], who took into consideration the $\mathrm{SUV}_{\text {max }}$ at FDG-PET before therapy in a series of 44 patients affected by synovial sarcoma and found that pre-therapy $\mathrm{SUV}_{\max }$ was predictive for overall survival and progression-free survival. Furthermore, patients with $\mathrm{SUV}_{\text {max }}$ greater than 4.35 at diagnosis had a decreased disease-free survival and were therefore at risk for having focal recurrences and metastatic disease. It was also taken into consideration as a prognostic factor the result of the FDG-PET carried out after the neo-adjuvant chemotherapy.

Sato et al. [6] evaluated a series of 13 patients by measuring the sarcoma $\mathrm{SUV}_{\max }$ before and after the neoadjuvant chemotherapy and correlated them with the expression of metastasis-related glycolytic enzyme and autocrine motility factor/phosphoglucose isomerase by immuno-histochemical examination in surgically excised tumours. The follow-up was 4 years. They found that mean SUV before therapy was similar in patients with and without metastasis, while mean SUV after therapy was significantly lower in those without metastasis. Furthermore, this last parameter was significantly correlated with the presence of glycolytic enzyme and autocrine motility factor/phosphoglucose isomerase.

\section{PET and the evaluation of response to therapy}

In case neo-adjuvant chemotherapy is indicated, the noninvasive evaluation of the response to therapy is very difficult with standard methods since the mass size usually doesn't change much even in responder patients. 
Evilevitch et al. [7] found that the decrease of SUV after neo-adjuvant chemotherapy is strongly correlated to the pathological response to therapy. They evaluated a series of 42 patients with resectable biopsy-proven soft-tissue sarcomas who underwent an FDG-PET before and after therapy, and found that in histo-pathological responders, the reduction of FDG uptake was significantly greater than in nonresponders. Using a $60 \%$ decrease in tumour FDG uptake as a threshold, the sensitivity was $100 \%$ and the specificity was $71 \%$ for assessment of histopathological response.

Ye et al. [8] did the same on a group of 15 patients affected by osteosarcoma and found that it was possible to discriminate between responders and non-responders on the basis of PET results, but that the measurement of TBR (tumour-tobackground ratio) was significantly better than SUV.

This result, however, needs to be confirmed, since the interobserver variability of TBR seems to be very high according to research by Benz et al. [9]. They evaluated which method was the most effective in measuring the response to therapy on PET images and found that $S_{U V} V_{\max }$ and $\mathrm{SUV}_{\text {peak }}$, not TBR, provided the most robust measurements of glucose metabolism in sarcomas.

\section{PET to stage and restage sarcomas}

The good accuracy of FDG-PET for staging and restaging malignancies suggested the idea to test this imaging modality also for patients affected by sarcomas. Furthermore, PET is a single-step exam allowing the evaluation of the whole body, and it's not difficult to include in the field of view even the extremities if needed.
This was mainly for bone sarcomas, and therefore most of the patient populations taken into consideration in published literature are paediatrics. All the studies are designed comparing the result of a FDG-PET scan to conventional imaging modalities (MR, CT, bone scintigraphy, US and chest radiography) in order to assess the accuracy of PET in determining the extension of disease both at staging and re-restaging.

Völker et al. [10] evaluated 46 paediatric patients affected by Ewing, Osteo or Rhabdomyosarcoma in a multicenter study. All the patients underwent a FDG-PET for staging the disease and its result was compared to standard imaging procedures. FDG-PET and conventional imaging were equally accurate for detecting the primary tumour (100\% accuracy), PET was superior in assessing lymph node involvement and bone manifestations while CT turned out to be more accurate for the detection of pulmonary metastasis. So, it turned out that FDG-PET has a very significant impact on the management of paediatric sarcomas compared to conventional imaging.

Similar results were obtained by Tateishi et al. [11] on a patient population of 117 patients. They found a very high accuracy in defining the exact TNM (more than 90\%) when FDG-PET/CT was combined with conventional imaging results.

Sarcoma recurrence can also be successfully and easily demonstrated with FDG-PET in paediatric patients. This was demonstrated by Arush et al. [12] on a series of 19 patients, who presented a FDG-PET scan that helped in the correct interpretation of conventional imaging findings and was able to detect otherwise unknown metastasis in two patients.
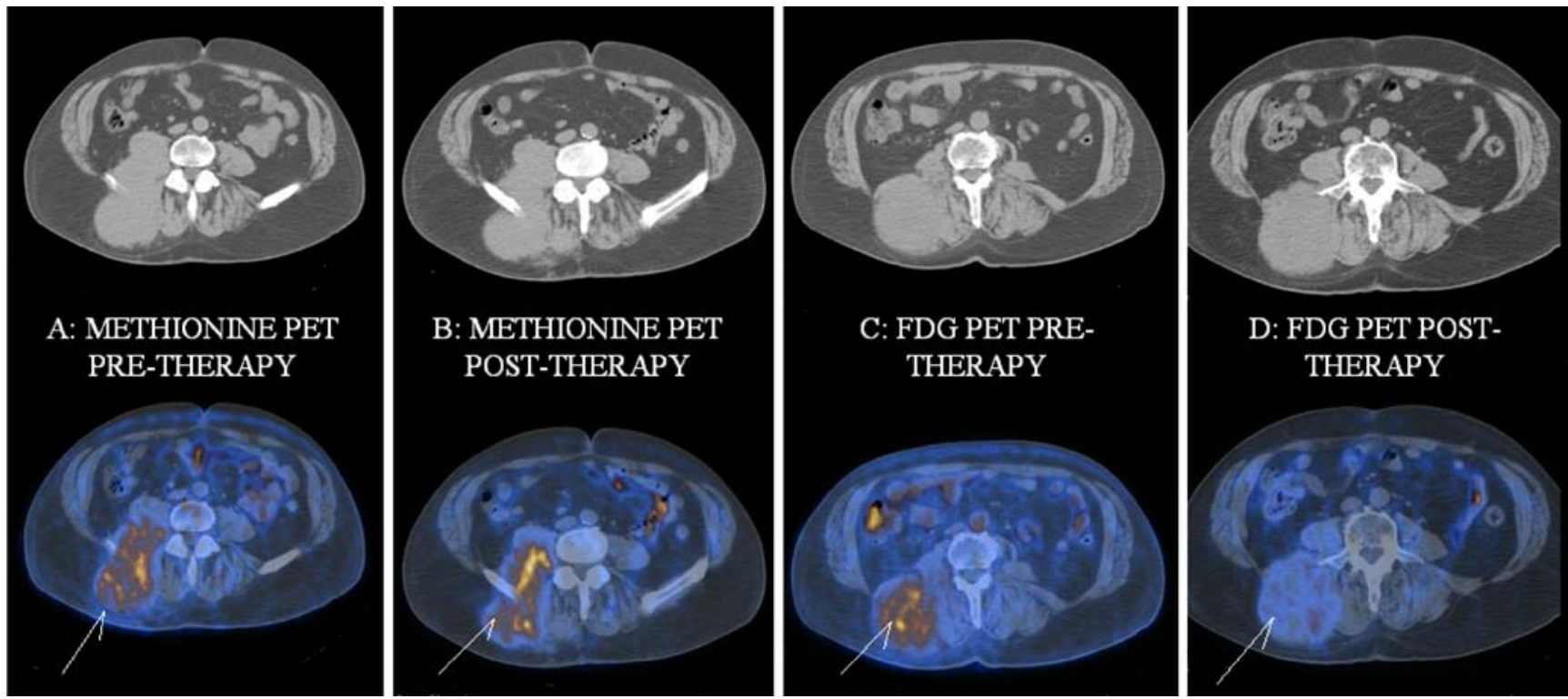

Fig. 1 Patient affected by synovial monofasic sarcoma of the right gluteus (arrows). Methionine PET/CT and FDG-PET/CT carried out before and after neo-adjuvant chemotherapy. FDG better demonstrates the response to therapy in comparison to methionine 


\section{Sarcomas and PET: other tracers}

FDG proved to be accurate and sensitive for the evaluation of soft-tissue and bone sarcoma since the major part of those neoplastic diseases are intermediate or high-grade tumours.

Some groups, however, tested different radiopharmaceuticals to understand if there may be somehow an added value in comparison to FDG.

Buck et al. [13], for example, evaluated the role of FLTPET to differentiate benign from malignant tumours (FLT is a marker of proliferation) and to detect manifestation sites of bone and soft-tissue sarcomas. Their patient population consisted of 22 patients with established or suspected bone or soft-tissue lesions. FLT-PET was compared to FDG-PET, MR, CT and biopsy. They found a significant correlation between FLT uptake and tumour grade at biopsy, which was not found for FDG, and it was concluded that FLT-PET is a suitable imaging procedure for evaluating soft-tissue and bone tumours.

Another interesting study by Tateishi et al. [14] is based on the use of choline-PET for staging 16 patients with softtissue and bone sarcoma, in comparison to bone scintigraphy, chest CT and MR. In this case, tumour staging was confirmed by histological examination and/or by an obvious progression in number and/or size of the lesions at follow-up examinations. The results were very positive for PET since it gave a correct result for the assessment of TNM in 15 out of 16 patients, while conventional imaging gave a correct TNM in 8 out of 16 patients. The major limit of this study, however, is the absence of data on FDG-PET.

Also methionine was evaluated for the detection and assessment of neo-adjuvant chemotherapy effect in combination with FDG in a preliminary study in nine patients by Ghigi et al. [15]. This study demonstrated that despite the fact that methionine is accurate in highlighting the primary mass, FDG is more suitable for distinguishing responder from non-responder patients since the difference between pre-therapy and post-therapy uptake is much greater for FDG in responder patients (Fig. 1).

\section{Conclusion}

According to the preliminary data so far published in the literature, PET seems to be a very valuable method for evaluating patients with soft-tissue and bone sarcomas. FDG seems to be the best tracer, but only a few papers are based on other compounds, so further studies are required before confirming this result.

FDG-PET seems to be useful in determining patient prognosis, for assessing the response to neo-adjuvant chemotherapy and in staging or re-staging the disease, and significantly contributes to the correct management of the patient. Based on the published data, it is possible to predict a wider employment of this imaging method in the future in patients affected by sarcomas.

\section{References}

1. Charest M. FDG PET-CT imaging in primary osseous and soft tissue sarcomas: a retrospective review of 212 cases. Eur J Nucl Med Mol Imaging. 2009; doi:10.1007/s00259-009-1203-0.

2. Skubitz KM, D'Adamo DR. Sarcoma. Mayo Clin Proc. 2007;82 (11):1409-32.

3. Toner GC, Hicks RJ. PET for sarcomas other than gastrointestinal stromal tumors. Oncologist. 2008;13(Suppl 2):22-6.

4. Eary JF, O'Sullivan F, O'Sullivan J, Conrad EU. Spatial heterogeneity in sarcoma $18 \mathrm{~F}-\mathrm{FDG}$ uptake as a predictor of patient outcome. J Nucl Med. 2008;49(12):1973-9.

5. Lisle JW, Eary JF, O'Sullivan J, Conrad EU. Risk assessment based on FDG-PET imaging in patients with synovial sarcoma. Clin Orthop Relat Res. 2009 Jun;467(6):1605-11.

6. Sato J, Yanagawa T, Dobashi Y, Yamaji T, Takagishi K, Watanabe H. Prognostic significance of $18 \mathrm{~F}-\mathrm{FDG}$ uptake in primary osteosarcoma after but not before chemotherapy: a possible association with autocrine motility factor/phosphoglucose isomerase expression. Clin Exp Metastasis. 2008;25(4):427-35.

7. Evilevitch V, Weber WA, Tap WD, Allen-Auerbach M, Chow K, Nelson SD, et al. Reduction of glucose metabolic activity is more accurate than change in size at predicting histopathologic response to neoadjuvant therapy in high-grade soft-tissue sarcomas. Clin Cancer Res. 2008;14(3):715-20.

8. Ye Z, Zhu J, Tian M, Zhang H, Zhan H, Zhao C, et al. Response of osteogenic sarcoma to neoadjuvant therapy: evaluated by $18 \mathrm{~F}$ FDG-PET. Ann Nucl Med. 2008;22(6):475-80.

9. Benz MR, Evilevitch V, Allen-Auerbach MS, Eilber FC, Phelps ME, Czernin J, et al. Treatment monitoring by $18 \mathrm{~F}-\mathrm{FDG}$ PET/CT in patients with sarcomas: interobserver variability of quantitative parameters in treatment-induced changes in histopathologically responding and nonresponding tumors. J Nucl Med. 2008;49(7):1038-46.

10. Völker T, Denecke T, Steffen I, Misch D, Schönberger S, Plotkin $\mathrm{M}$, et al. Positron emission tomography for staging of pediatric sarcoma patients: results of a prospective multicenter trial. J Clin Oncol. 2007;25(34):5435-41.

11. Tateishi U, Yamaguchi U, Seki K, Terauchi T, Arai Y, Kim EE. Bone and soft-tissue sarcoma: preoperative staging with fluorine 18 fluorodeoxyglucose PET/CT and conventional imaging. Radiology. 2007;245(3):839-47.

12. Arush MW, Israel O, Postovsky S, Militianu D, Meller I, Zaidman I, et al. Positron emission tomography/computed tomography with 18fluoro-deoxyglucose in the detection of local recurrence and distant metastases of pediatric sarcoma. Pediatr Blood Cancer. 2007;49(7):901-5.

13. Buck AK, Herrmann K, Büschenfelde CM, Juweid ME, Bischoff $\mathrm{M}$, Glatting $\mathrm{G}$, et al. Imaging bone and soft tissue tumors with the proliferation marker [18F]fluorodeoxythymidine. Clin Cancer Res. 2008;14(10):2970-7.

14. Tateishi U, Yamaguchi U, Maeda T, Seki K, Terauchi T, Kawai A, et al. Staging performance of carbon-11 choline positron emission tomography/computed tomography in patients with bone and soft tissue sarcoma: comparison with conventional imaging. Cancer Sci. 2006;97(10):1125-8.

15. Ghigi G, Micera R, Maffione AM, Castellucci P, Cammelli S, Ammendolia I, et al. 11C-Methionine vs 18F-FDG PET in soft tissue sarcoma patients treated with neoadjuvant therapy: preliminary results. In Vivo. 2009;23(1):105-10. 